\title{
ÉTUDE DU CARACTÈRE CULARD
}

\author{
V. - EXPÉRIENCE DE CROISEMENTS ENTRE BOVINS CULARDS ET NORMAUX
}

\author{
B. LOGEAY et B. VISSAC
}

avec la collaboration technique de B. Perreau

Station de Génétique quantitative et appliquée, Centre national de Recherches zootechniques, 78 -Jouy-en-Josas

Institut national de la Recherche agronomique

\section{RÉSUMÉ}

Dans un plan de croisement factoriel entre géniteurs culards (C) et normaux (N) des deux sexes appartenant à la race charolaise et entretenus dans des conditions de milieu identiques, les produits obtenus pouvaient être rangés dans l'une de ces deux catégories culards ou normaux, peu d'entre eux présentant un phénotype intermédiaire. Les pourcentages de produits culards étaient respectivement de 87,$5 ; 10 ; 9,5 ; 0$ dans les accouplements : $C \times C ; C \times N ; N \times C$; $N \times N$. Il semble, par ailleurs, que la note exprimant le caractère culard est en moyenne légèrement plus élevée dans le cas des veaux issus d'accouplements $\mathrm{C} \times \mathrm{N}$ et $\mathrm{N} \times \mathrm{C}$ que dans le cas des veaux issus de parents normaux. Ces résultats font penser à une action monofactorielle à dominance partielle et à pénétrance incomplète, qui serait au minimum de $87 \mathrm{p} .100 \mathrm{chez}$ les homozygotes culards et comprise entre 10 et 20 p. 100 chez les hétérozygotes.

\section{INTRODUCTION}

L,e caractère culard a fait l'objet de nombreuses recherches qui se sont surtout attachées à donner en premier lieu une description morphologique et anatomique de ces animaux puis à étudier leurs caractéristiques physiologiques et leurs performances zootechniques. Les principales observations ont été effectuées par KaIser (I888), PACi (r935), RaImondi (I957) et Hanset (I96I).

Les différentes hypothèses sur la transmission héréditaire du caractère ont généralement toutes un point commun : cette anomalie serait due à un gène autosomal. Mais pour WRIEDT (I929) travaillant sur la race Shorthorn, pour PACI (I935) qui réalisa 240 accouplements entre animaux piémontais normaux et culards, pour RAIMONDI (I957) le gène est dominant et son action peut être influencée par des gènes modificateurs et par le milieu. Par contre, en race Pie 
Noire (WEBER et IBSEN, I934 et ANONyme, I96I) et dans la race de Haute et Moyenne Belgique (HANSET, I96r) le gène serait récessif.

En I963, LAUVERGNE et al., essayent d'interpréter tous ces résultats d'après la théorie de 1'évolution de la dominance de Fisher (I958) : 1'allèle le plus avantageux tend à devenir dominant sur les autres allélomorphes sous 1'action de la sélection; les étapes intermédiaires de cette évolution correspondraient à l'action d'un gène à pénétrance incomplète et à expressivité variable. Il semble en effet d'une façon générale que le gène aurait tendance à être dominant dans les races à viande (piémontaise, Shorthorn) alors qu'il resterait récessif dans les races laitières (pie noire) et dans les races à viande où ce caractère est considéré comme gênant (Hereford et Angus aux U.S.A.).

Dès 1962 on a entrepris, en France, des recherches destinées à préciser le mode de transmission de ce caractère en race charolaise. Une enquête a été réalisée dans une trentaine d'élevages de cette race dans lesquels apparaissait fréquemment le caractère culard (DE LA GUERIVIère, I964; VISSAC et LAUVERGNE, I969). Simultanément on a étudié la descendance de taureaux charolais culards et normaux en liaison avec plusieurs centres d'insémination artificielle (ANoNymE, I966). Ces travaux ont fait apparaître les grandes variations d'expressivité du caractère culard avec 1'âge et les conditions d'élevage propres à chaque ferme tant chez les produits que chez les parents.

L'étude du déterminisme héréditaire du caractère culard nécessitait donc la réalisation d'une expérience sur des animaux élevés dans des conditions homogènes. La présente étude a pour objet de présenter et de discuter les résultats de cette expérience.

\section{I. - MATÉRIEL ET MÉTHODES}

\section{Schéma expérimental}

Les quatre types d'accouplement possibles entre animaux des deux sexes appartenant aux deux catégories de bovins normaux et culards ont été réalisés entre 1964 et $1968{ }^{(1)}$. On a choisi délibérément pour cette expérience des géniteurs mâles et femelles qui exprimaient ou non le caractère culard sans aucune ambiguïté possible. Dans ce but, trente génisses culardes nées en 1962 ont été mises en comparaison, dans deux élevages, avec des génisses normales de même âge et issues du propre troupeau de chaque éleveur. Dans le schéma initial d'accouplement les génisses de chaque troupeau devaient être saillies alternativement par un taureau normal et par un culard. En fait des problèmes matériels liés aux difficultés d'élevage des animaux culards nous ont amenés à modifier ce schéma.

Dans l'élevage $A$ les femelles culardes achetées en 1962 et saillies en 1963 par un seul taureau normal devaient vêler à deux ans. Cette tentative fut un échec puisque cinq génisses culardes sur quinze vêlèrent en 1964, alors que cette même année onze génisses normales sur dix-sept donnaient naissance à un veau. En 1964, toutes les vaches, quel que soit leur phénotype, furent saillies à nouveau par un taureau normal et dix culardes sur quatorze furent gestantes.

Cette même année les génisses normales et culardes de l'élevage $B$, achetées en 1963 à l'âge de 19-20 mois, furent inséminées pour la première fois avec de la semence de taureau culard. Seize génisses dont deux culardes seulement mirent bas. Pour des raisons pratiques liées aux difficultés de vêlage, toutes les vaches culardes gestantes furent rassemblées pendant l'hiver 1964-65 dans l'élevage $B$, tandis que les femelles vides étaient regroupées dans l'autre élevage.

Ces deux premières années d'expérience ont fait apparaître les nombreuses difficultés liées à l'exploitation des femelles présentant le caractère culard, dans les conditions d'élevage extensif

(1) Ces deux phénotypes parentaux seront désignés par $N$ et $C$ dans la suite de ce travail. L,e phénotype du père est placé en tête pour désigner un type d'accouplement. 
de la zone charolaise. Ces difficultés concernent la détection des chaleurs peu apparentes en général, la surveillance et la réalisation des mises bas, l'allaitement du veau enfin, la production laitière et l'instinct maternel des femelles culardes étant très limités (VIssac et al., 1970). Pour réaliser une surveillance très stricte du troupeau, toutes les vaches culardes furent rassemblées en 1965 à la ferme expérimentale de La Minière. Par ailleurs, le faible nombre de produits issus d'accouplement $C \times C$ obtenus à cette époque nous a conduits à n'inséminer à partir de 1966 les femelles culardes qu'avec de la semence de tauraux culards. Cette dernière partie de l'expérience fut réalisée sans troupeau témoin de femelles normales.

Au cours de l'expérience, cinq taureaux dont quatre culards ont été utilisés. L'influence du mâle va donc être importante sur l'ensemble des résultats, mais elle ne pourra être analysée valablement puisqu'en général un type d'accouplement donné n'a été réalisé qu'avec un seul taureau.

Les différentes phases à considérer dans le déroulement de cette expérimentation sont schématisées sur le tableau 1. Ce tableau comporte notamment le phénotype des taureaux utilisés chaque année dans chaque troupeau. La liste des mâles ainsi que le nombre de veaux observés par type d'accouplement et par année frgurent par ailleurs sur le tableau 2.

TABLEAU I

Déroulement de l'expérience

\begin{tabular}{|c|c|c|c|c|c|}
\hline & Année & & & Élevage $A$ & Élevage $B$ \\
\hline $\begin{array}{l}1962 \\
1963 \\
1964 \\
\text { Hiver } \\
\text { r965 }\end{array}$ & 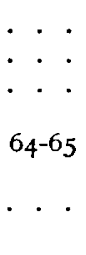 & $\begin{array}{l}\cdot \\
\cdot \\
\cdot\end{array}$ & $\begin{array}{l}\cdot . \\
. \\
. \\
. \\
.\end{array}$ & $\begin{array}{c}\text { Achat de I8 génisses âgées de } 6 \text { mois } \\
\text { Saillie }\left(N_{1}\right) \\
\text { Vêlage } \\
\text { Saillie }\left(N_{1}\right) \\
\text { Regroupement des } q \text { culardes vides } \\
\text { (A et B) } \\
\text { Vêlage des normales } \\
\text { Saillie }\left(N_{1}\right)\end{array}$ & $\begin{array}{l}\text { Achat de génisses âgées de } 18 \text { mois } \\
\text { Saillie }\left(C_{1}\right) \\
\text { Regroupement des }+ \text { culardes ges- } \\
\text { tantes (A et B) } \\
\text { Vêlage des culardes et normales } \\
\text { Saillie }\left(C_{2}\right)\end{array}$ \\
\hline $\begin{array}{l}\text { Hiver } \\
\text { r966 } \\
\text { r967 }\end{array}$ & $\begin{array}{c}\cdot \cdot \cdot \\
\cdot . \cdot \\
\cdot . \\
\cdot\end{array}$ & & . & \multicolumn{2}{|c|}{$\begin{array}{l}\text { Regroupement de toutes les } q \text { o culardes à l'étable expérimentale de La } \\
\text { Minière } \\
\qquad \begin{array}{l}\text { Vêlage } \\
\text { Saillie }\left(C_{3}\right) \\
\text { Vêlage } \\
\text { Saillie }\left(C_{2}, C_{4}\right) \\
\text { Vêlage }\end{array}\end{array}$} \\
\hline
\end{tabular}

N.-B. : Les lettres $N$ et $C$ indiquent que le taureau utilisé pour la saillie ou l'insémination était normal ou culard, l'indice désigne le taureau lui-même.

\section{Conditions d'élevage}

Dans les exploitations $A$ et $B$, les animaux étaient élevés dans les conditions normales de la zone charolaise : les vaches passaient l'hiver en stabulation entravée et recevaient une alimentation composée de fourrages grossiers. Après le vêlage, au début du printemps, elles étaient mises au pâturage durant tout l'été et l'automne. Les veaux étaient allaités par leur mère et recevaient un complément 'd'aliments concentrés.

A la ferme expérimentale de La Minière les vaches reçurent le même type d'alimentation que précédemment, mais les veaux étaient séparés de leurs mères qui étaient traites, et ils furent allaités artificiellement. Cette façon de faire a permis de réduire l'incidence du régime alimentaire du veau, lié au potentiel laitier de sa mère, sur l'expression du caractère culard.

\section{Appréciation du caractère culard}

On sait que cette appréciation est rendue difficile par l'absence de mesure objective ainsỉ que par l'évolution de diverses caractéristiques morphologiques du bovin culard soit avec l'état sanitaire et les conditions nutritionnelles, soit avec l'âge de l'animal : ainsi l'hypertrophie mus- 
TABLEAU 2

Effectif de femelles et nombre de vêlages par génotype et par année

\begin{tabular}{|c|c|c|c|c|c|c|}
\hline \multirow{3}{*}{$\begin{array}{l}\text { Année } \\
\text { de } \\
\text { vêlage }\end{array}$} & \multirow{2}{*}{\multicolumn{2}{|c|}{ Taureaux utilisés }} & \multicolumn{4}{|c|}{ Femelles } \\
\hline & & & \multicolumn{2}{|c|}{ Culardes } & \multicolumn{2}{|c|}{ Normales } \\
\hline & Type & Nom & $\begin{array}{l}\text { Nombre } \\
\text { de } \\
\text { femelles }\end{array}$ & $\begin{array}{l}\text { Nombre } \\
\text { de vêlages } \\
\text { consécutifs }\end{array}$ & $\begin{array}{c}\text { Nombre } \\
\text { de } \\
\text { femelles }\end{array}$ & $\begin{array}{l}\text { Nombre } \\
\text { de vêlages } \\
\text { consécutifs }\end{array}$ \\
\hline 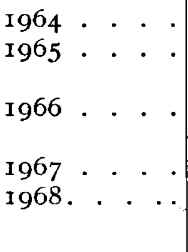 & $\begin{array}{l}N_{1} \\
N_{1} \\
C_{1} \\
N_{1} \\
C_{2} \\
C_{3} \\
C_{2} \\
C_{4}\end{array}$ & $\begin{array}{l}\mathrm{X} \ldots \\
\mathrm{X} \ldots \\
\text { Sirius } \\
\mathrm{X} \ldots \\
\text { Orient } \\
\text { Titus } \\
\text { Orient } \\
\text { Vélo }\end{array}$ & $\begin{array}{l}\text { I5 } \\
\text { I4 } \\
\text { I } 4 \\
16 \\
\text { I } \\
22 \\
\text { I0 }\end{array}$ & $\begin{array}{r}5 \\
10 \\
2 \\
2 \\
14 \\
3 \\
21 \\
10\end{array}$ & $\begin{array}{l}\text { I7 } \\
\text { I4 } \\
\text { I } 4\end{array}$ & $\begin{array}{l}\text { II } \\
\text { II } \\
\text { I } 4\end{array}$ \\
\hline Total. . . . & $\begin{array}{l}\text { Normat } \\
\text { Culards }\end{array}$ & & $\begin{array}{l}45 \\
58\end{array}$ & $\begin{array}{l}29 \\
36\end{array}$ & $\begin{array}{l}31 \\
r_{4}\end{array}$ & $\begin{array}{l}22 \\
14\end{array}$ \\
\hline
\end{tabular}

TABLEAU 3

Répartition des pointages par caractère en fonction des génotypes parentaux.

\begin{tabular}{|c|c|c|c|c|c|c|c|c|c|c|c|c|}
\hline Génotype & \multicolumn{3}{|c|}{$C \times C$} & \multicolumn{3}{|c|}{$C \times N$} & \multicolumn{3}{|c|}{$N \times C$} & \multicolumn{3}{|c|}{$N \times N$} \\
\hline $\begin{array}{l}\text { critères } \\
\text { morphologiques }\end{array}$ & 0 & $\mathbf{I}$ & 2 & o & $\mathbf{I}$ & 2 & o & $\mathbf{I}$ & 2 & o & I & 2 \\
\hline $\begin{array}{l}\text { I. Macroglossie et brachygna- } \\
\text { thisme }\end{array}$ & 16 & 0 & 0 & T & 0 & 0 & 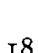 & 0 & 0 & 78 & 0 & \\
\hline 2. Musculature de 1'épaule. . & o & 2 & I4 & $\begin{array}{r}4 \\
7\end{array}$ & 5 & 2 & 8 & Io & o & I5 & 3 & o \\
\hline 3. Compacité générale . . . & o & 4 & 12 & 6 & 5 & 3 & 7 & 8 & 3 & 12 & 6 & o \\
\hline 4. Défectuosités d'aplombs. & I I & 4 & I & 14 & o & 0 & I6 & 2 & o & I 7 & $\mathbf{I}$ & o \\
\hline 5. Rétraction du ventre . . & 2 & 7 & 7 & $\mathrm{r} 4$ & o & o & I6 & 2 & o & 17 & I & o \\
\hline 6. Inclinaison $d u$ bassin . & I & 5 & ro & 8 & 3 & 3 & 3 & I 5 & o & Io & 6 & 2 \\
\hline $\begin{array}{l}\text { 7. Attache de la queue } . ~ \\
\text { 8. Rebondi musculaire de la }\end{array}$ & 2 & 5 & 9 & II & 3 & o & 7 & I I & o & I3 & 5 & o \\
\hline 9. Saillies musculaires géné- & 0 & 3 & I3 & 9 & 3 & 2 & 2 & I3 & 3 & 8 & 8 & 2 \\
\hline rales. . . . . . . . . : & o & 3 & 13 & Io & 3 & I & 4 & Io & 4 & I7 & 7 & o \\
\hline Io. Finesse de l'os . . . . . & 0 & 5 & I I & 8 & 5 & I & 7 & Io & I & 4 & I3 & I \\
\hline
\end{tabular}


culaire souvent peu apparente à la naissance est en général très accusée à partir de 3 mois chez les animaux en bonne santé et nourris ad libitum; par contre la macroglossie et les déformations osseuses des membres qui se manifestent chez les animaux culards à la naissance ont pratiquement disparu à trois mois (Vissac et al., 1970).

Dans cette étude, le caractère culard a été apprécié globalement entre la naissance et trois mois pour chaque veau en tenant compte des variations d'expressivité que nous venons de rappeler. On a par ailleurs utilisé la table de pointage proposée par Neuvy et Vissac (1962). Rappelons que cette table (tabl. 3) concerne dix critères morphologiques associés en général chez les animaux culards et qui ont trait à l'hypertrophie musculaire, à la morphologie particulière du train postérieur et aux défectuosités rappelées ci-dessus. Chaque critère était noté : 0 (phénotype normal), 1 (phénotype intermédiaire) et 2 (phénotype culard). Ce pointage était réalisé à 3 mois, 6 mois et 9 mois. On a utilisé dans cette étude la moyenne des notes d'un animal pour ces trois âges. La somme correspondant aux dix notes ainsi obtenues, et qui peut varier de 0 à 20 , fournit une deuxième appréciation du caractère culard; les animaux dont la somme des notes est supérieure à 12 sont en général considérés comme culards dans le jugement global; les quelques exceptions concernent des animaux en mauvaise santé. La différence d'effectifs des veaux soumis à ces deux appréciations s'explique par la disparition d'un certain nombre d'animaux entre la naissance et 3 mois, âge auquel a été effectué le premier pointage détaillé. Toutes ces opérations ont été réalisées par le même opérateur de 1964 à 1968.

Au cours de cette période, 156 vaches normales ou culardes furent mises à la reproduction, 109 mirent bas $(68 \%), 83$ veaux vivants furent appréciés globalement et 66 firent l'objet d'au moins un pointage.

\section{II. - RÉSULTATS EXPÉRIMENTAUX}

On a représenté sur le tableau 3 la répartition des notes par caractère pour les produits issus de chaque type d'accouplement. L'examen de ces chiffres confirme le fait que les défectuosités correspondant aux notes $\mathrm{n}^{\mathrm{os}}$ I et 4 ont pratiquement disparu sur l'ensemble des veaux aux âges où sont réalisés les pointages.

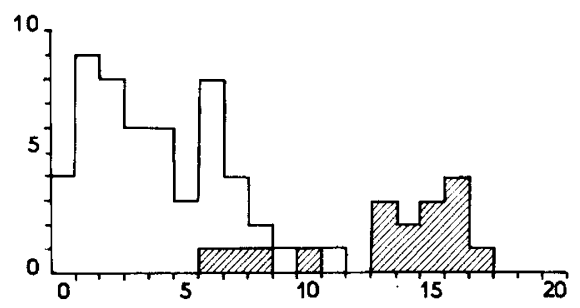

FIG. I. - Distribution de fréquence des notes de pointage suivant l'appréciation globale des veaux. En ordonnée les effectits réels.

Wh veaux culards.

$\square$ veaux normaux.

En ce qui concerne la somme des ro notes on observe une répartition de l'échantillon global des veaux en 2 catégories pratiquement distinctes de part et d'autre des notes II-I2. Ces deux catégories correspondent en fait, trois veaux en mauvaise santé mis à part, à la répartition entre veaux normaux et culards effectuée globalement à 3 mois ou plus précocement (fig. I).

La comparaison des 4 types d'accouplement réalisés permet d'observer que les 16 veaux pointés dont les deux parents sont culards ont, tous sauf 3 , une note de pointage supérieure à $\mathrm{I} 2$ qui permet de les considérer comme " culards " (fig. 2). Parmi les trois veaux dont la note est inférieure à 12 , deux sont en fait des culards en mauvaise santé, le troisième présente toutes les caractéristiques d'un animal normal. L'appréciation globale réalisée sur 24 veaux (tabl. 4), dont 
les I6 pointés, confirme l'apparition de descendants non culards à la suite d'accouplements $C \times C$ (3 produits étaient absolument normaux, y compris celui ayant un pointage inférieur à I2) la probabilité d'erreurs de paternité portant sur les 3 animaux est en effet négligeable. Au contraire, les veaux issus des trois autres types d'accouplement avaient tous une note de pointage inférieure à $\mathrm{Ir}$ bien que deux d'entre eux, issus d'accouplements $C \times N$ et $N \times C$ doivent être considérés comme des culards. Ces résultats sont eux aussi en concordance avec ceux relatifs à l'appréciation globale ; on a pu observer en effet deux culards seulement sur 20 et 2 I veaux pour chaque catégorie d'accouplements $C \times N$ et $N \times C$ (tabl. 4).

TABIEAU 4

Descendance des différents types d'accouplements entre normaux et culards (Appréciation globale)

\begin{tabular}{|c|c|c|c|c|}
\hline \multicolumn{2}{|c|}{$\begin{array}{l}\text { Génotypes } \\
\text { parentaux }\end{array}$} & \multirow{2}{*}{$\begin{array}{c}\text { Nombre de veaux } \\
\text { culards }\end{array}$} & \multirow{2}{*}{$\begin{array}{c}\text { Nombre de veaux } \\
\text { normaux }\end{array}$} & \multirow{2}{*}{ de culards } \\
\hline$d 0$ & 우 & & & \\
\hline $\begin{array}{l}C \\
C \\
N \\
N\end{array}$ & $\begin{array}{l}C \\
N \\
C \\
N\end{array}$ & $\begin{array}{r}2 \mathrm{I} \\
2 \\
2 \\
0\end{array}$ & $\begin{array}{r}3 \\
18 \\
19 \\
18\end{array}$ & $\begin{array}{l}87,5 \\
\text { ro } \\
9,5 \\
0\end{array}$ \\
\hline Total & . & 25 & $5^{8}$ & $30, \mathbf{I}$ \\
\hline
\end{tabular}

La comparaison statistique des notes moyennes de pointage correspondant aux quatre types d'accouplement pris 2 à 2 permet enfin de conclure que les veaux issus de parents culards se différencient très nettement des trois autres catégories. Les pointages moyens des veaux issus d'accouplements $C \times N$ et $N \times C$ sont par ailleurs homogènes et supérieurs (différence significative au seuil $5 \%$ dans le deuxième cas) au pointage moyen des veaux issus de parents normaux (tabl. 5). La différence subsiste lorsqu'on compare les pointages moyens de l'ensemble des veaux normaux issus d'accouplements $C \times N$ et $N \times C$ à celui des veaux fournis par des parents normaux. Néanmoins cette différence n'est pas significative ( $t=\mathrm{I}, 43$ pour 46 degrés de liberté).

Étant donné d'une part le faible nombre de taureaux utilisés dans cette expérience, d'autre part les troubles de reproduction des femelles présentant le caractère culard, il ne nous a pas été possible de faire une analyse de la variabilité génétique entre descendances de parents appartenant à un même génotype. Nous noterons cependant que les trois veaux normaux issus d'accouplements $C \times C$ sont fils de deux taureaux ayant eu chacun cinq veaux; les deux autres géniteurs mâles culards n'ont eu que des veaux culards, respectivement 2 et I 2 
TABLEAU 5

Comparaison des valeurs moyennes de pointage des veaux en fonction des génotypes parentaux (Tests de Student-Fisher)

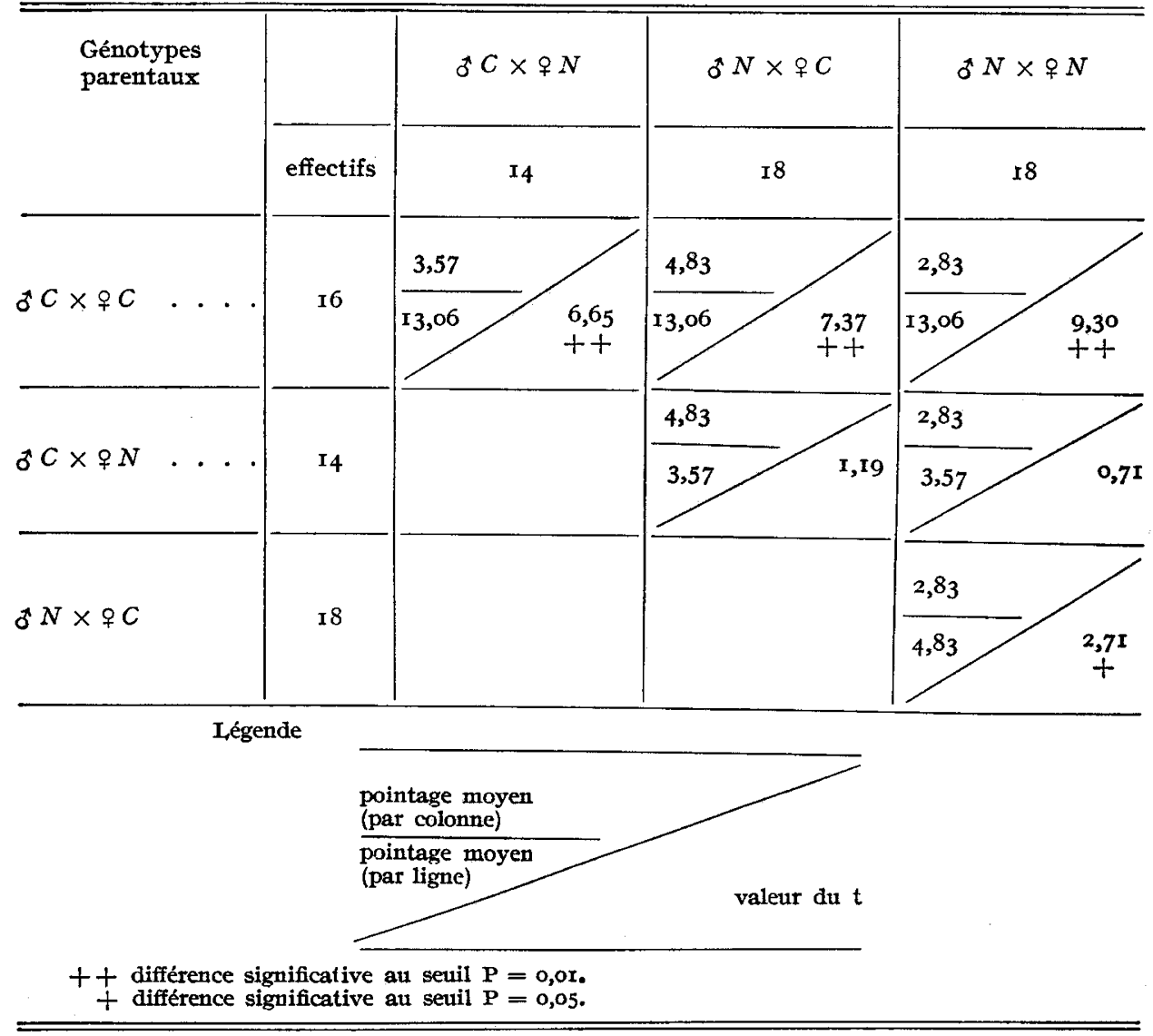

TABIEAU 6

Répartition des descendances par taureau dans les accouplements $C \times C$ (appréciation globale)

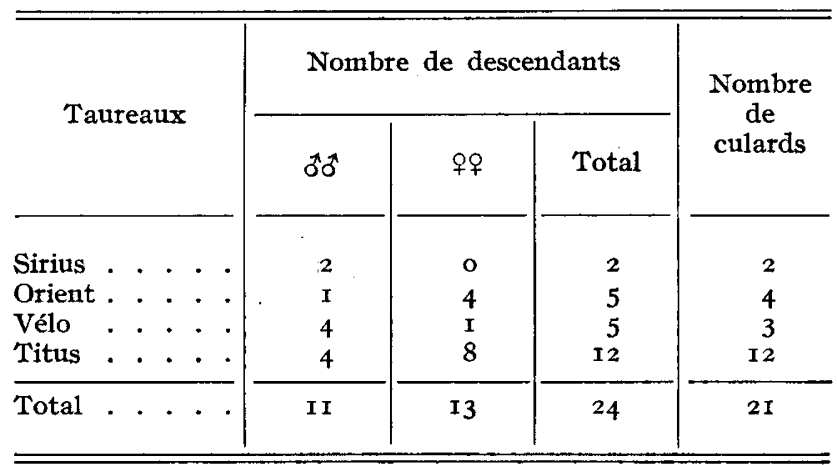


(tabl. 6). Les veaux normaux issus d'accouplements $C \times C$ proviennent enfin de trois femelles différentes.

La répartition par sexe des veaux correspondant aux quatre types d'accouplement ne s'écarte de la normale (50\%) pour aucun d'entre eux (tabl. 7).

TABIEAU 7

Répartition par sexe des veaux nés, quel que soit leur phénotype (appréciation globale)

\begin{tabular}{|c|c|c|c|c|}
\hline \multicolumn{2}{|c|}{ Type d'accouplement } & \multirow{2}{*}{$\begin{array}{c}\text { Nombre de } \\
\delta^{*} \delta^{*}\end{array}$} & \multirow{2}{*}{$\begin{array}{c}\text { Nombre de } \\
+q\end{array}$} & \multirow{2}{*}{$\chi^{2}$} \\
\hline$\sigma^{\circ} \sigma^{\circ}$ & 아 & & & \\
\hline C & $C$ & $\mathrm{r}_{5}$ & I6 & 0,02 \\
\hline$C$ & $N$ & 8 & I I & 0,46 \\
\hline$N$ & C & 12 & I 5 & 0,33 \\
\hline$N$ & $N$ & I 2 & Io & $0, \mathbf{I} 8$ \\
\hline \multicolumn{2}{|l|}{ Total } & 47 & $5^{2}$ & 0,25 \\
\hline
\end{tabular}

\section{III. - DISCUSSION ET CONCLUSION}

La distribution de fréquence du pointage culard correspond dans son ensemble à celles observées dans les enquêtes en ferme réalisées dans la zone charolaise (DE LA GUÉRIVIÈRE, I964) et dans la zone d'action des centres d'insémination artificielle utilisant des taureaux culards pour le croisement industriel (ANONYME, I966). On observe toutefois une distinction beaucoup plus tranchée entre les deux types de veaux, normaux et culards, ce qui confirme l'importance des variations d'expressivité du caractère dans les enquêtes en ferme et l'utilité de la présente recherche. Il convient de préciser que dans les études antérieures les auteurs se sont limités à distinguer les individus en deux catégories, seuls WRIEDT (r929) et RAIMONDI (I957) considérant les animaux à phénotype intermédiaire. La distinction nette entre deux classes d'animaux confirmerait en tout cas au premier abord l'hypothèse d'action d'un gène majeur émise par la plupart des auteurs.

En ce qui concerne le mode d'action de ce gène, nous avons comparé les résultats de cette étude à ceux obtenus antérieurement pour les mêmes types d'accouplements (tabl. 8). Si l'on excepte les résultats de l'étude de Paci (I935), ils sont tous remarquablement concordants. Les deux conceptions classiques de récessivité et de dominance simples de ce gène doivent tout d'abord être écartées: la première est en contradiction avec l'existence de quelques descendants normaux issus d'accouplements entre parents culards; la deuxième ne peut pas expliquer la fraction insuffisamment élevée de produits culards dans les croisements entre géniteurs de phénotypes différents. Il est par ailleurs intéressant 
CROISEMENTS ENTRE BOVINS CULARDS ET NORMAUX

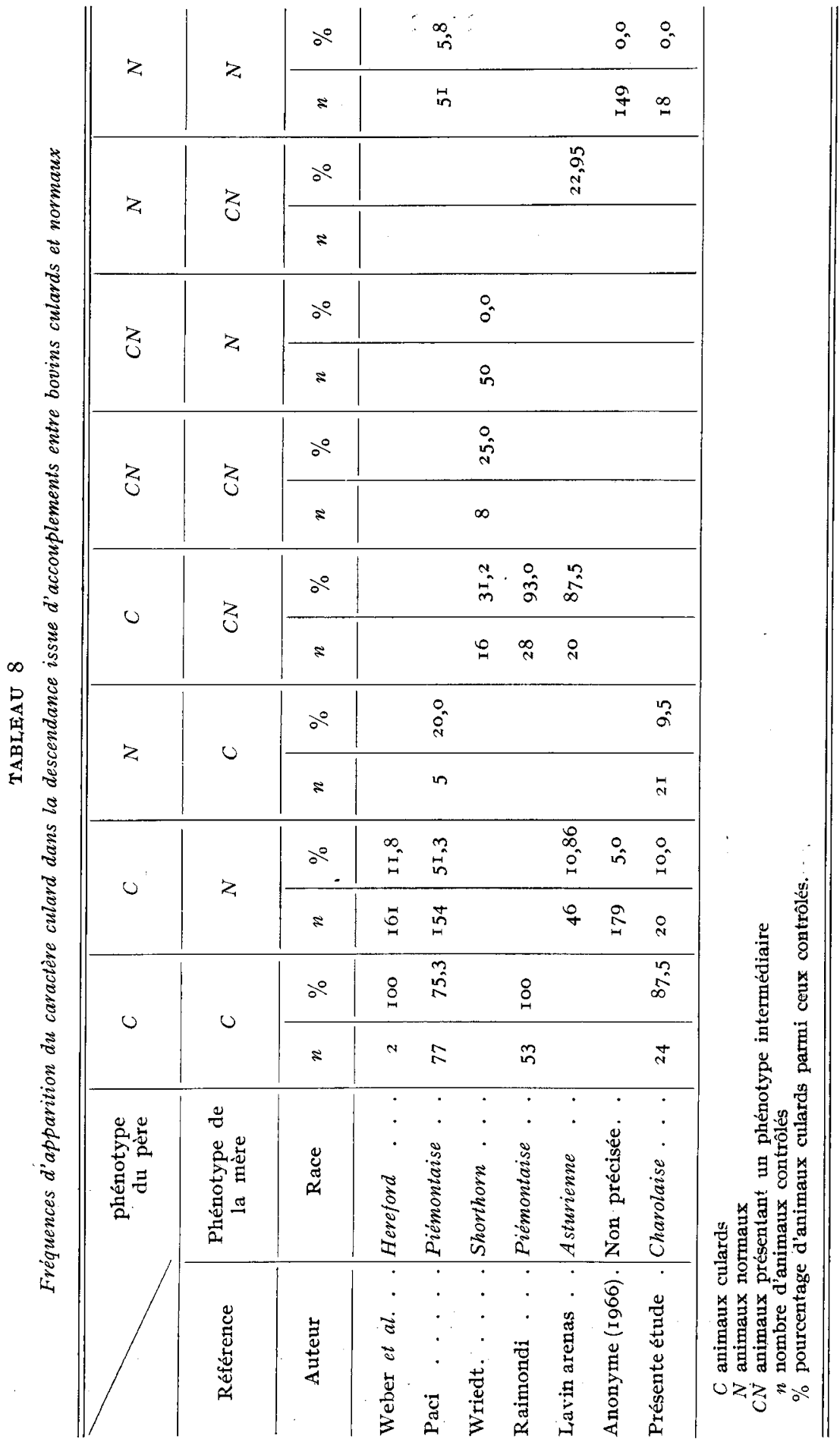




\section{a) $\mathrm{C} \times \mathrm{C}$}

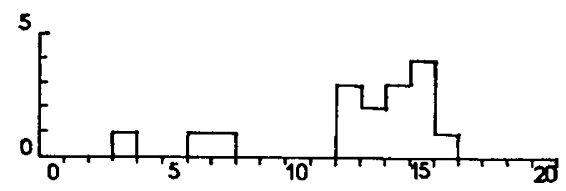

b) $\mathrm{C} \times \mathrm{N}$ et $\mathrm{N} \times \mathrm{C}$

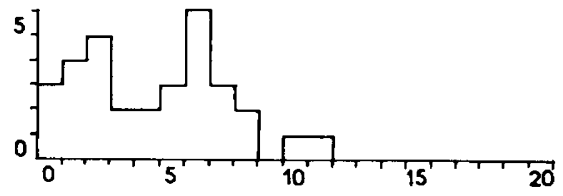

c) $\mathrm{N} \times \mathrm{N}$

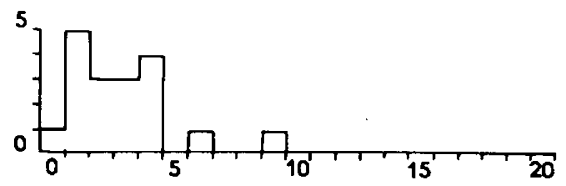

FIG. 2. - Distribution de fréquence des notes de pointage des veaux suivant les génotypes parentaux. En ordonnée les effectifs réels.

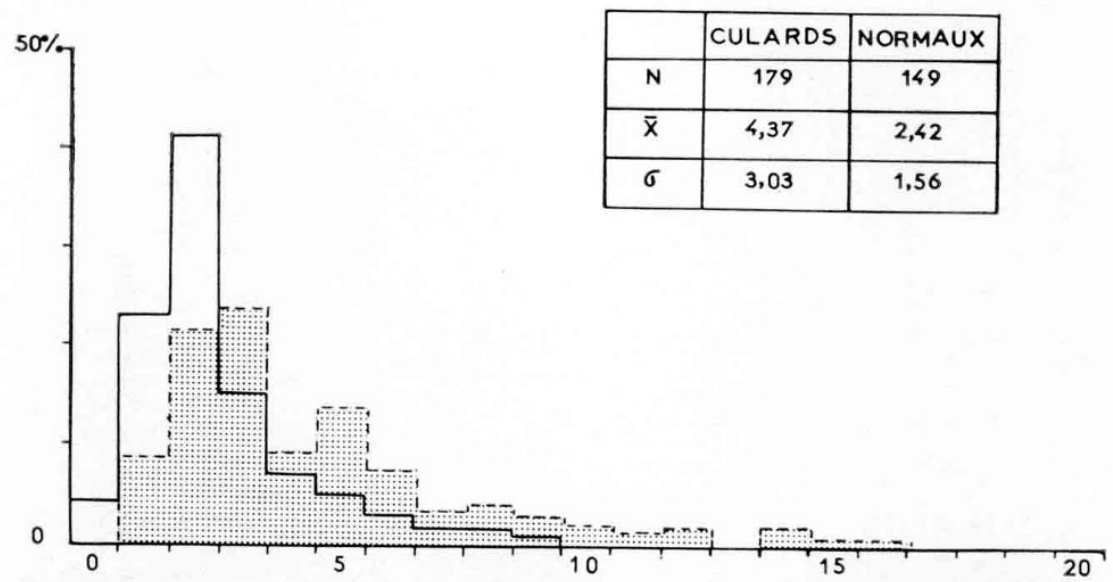

FIG. 3. - Distribution de fréquence des notes de pointage culard des descendants de taureaux culards et normaux. (Zone du Centre d'Insémination Artificielle de SOUAI-1964) (d'après Anonyme, 1966).

㵛 descendants de taureaux culards.

$\square$ descendants de taureaux normaux.

de constater que le pointage moyen des veaux normaux issus d'accouplements $N \times C$ ou $C \times N$ est plus élevé que celui des veaux issus de parents normaux (fig. 2). Cette observation a également été réalisée sur des résultats d'une autre étude (ANONyme, I966) portant sur un effectif plus important et où la descen- 
dance de six taureaux culards était comparée à celle de six taureaux normaux de race charolaise accouplés les uns et les autres à un échantillon de femelles de races rustiques et laitières : les veaux vraiment culards étant exclus, les distributions de fréquence des deux types de descendants étaient sensiblement décalées (fig. 3). On peut donc admettre, comme le pensent Rolinss et al. (I968), et dans l'hypothèse de monofactorialité que le génotype hétérozygote présente un phénotype intermédiaire entre le culard et le normal mais plus proche de ce dernier. D'après Roliriss, les notes o et I étant attribuées respectivement au culard et au normal, 1'hétérozygote exprimerait le caractère avec la note 0,75 . Cela reviendrait en définitive à postuler une action monofactorielle avec dominance partielle. Cette conclusion est en fait théorique car, si elle est retenue, 1'hétérozygote ne peut, de toute façon, être distingué de l'homozygote normal.

Une action monofactorielle simple ne pouvant être retenue on est donc conduit à envisager soit la récessivité à pénétrance incomplète soit la dominance à pénétrance incomplète, la pénétrance étant définie comme la fréquence avec laquelle un gène ou un groupe de gènes se manifeste extérieurement chez les individus le possédant. N'ayant pas d'élément suffisant pour conclure en faveur de l'une ou l'autre hypothèse, la deuxième ayant été retenue par d'autres auteurs (HANSET, I967) nous pouvons estimer un ordre de grandeur de la pénétrance du gène dans cette situation. D'après les résultats d'accouplements entre bovins culards mâles et femelles elle serait au minimum de 87 p. Ioo chez l'homozygote, quel que soit le génotype des reproducteurs utilisés. D’après les résultats d'accouplements $C \times N$ et $N \times C$, en supposant les bovins normaux homozygotes, on peut estimer la valeur de cette pénétrance chez l'hétérożygote entre ro $\mathrm{p}$. Ioo (cas où tous les géniteurs culards utilisés seraient homozygotes) et $20 \mathrm{p}$. Ioo (cas où ils seraient tous hétérozygotes). Ces chiffres peuvent être comparés à ceux fournis par HANSET (I967) en race de Haute et Moyenne Belgique : cet auteur estime la pénétrance du gène à $8 \mathrm{p}$. Ioo chez les hétérozygotes et à 60 p. Ioo chez les homozygotes.

En ce qui concerne les variations enregistrées entre les résultats des chercheurs pour un même type d'accouplement on peut les interpréter soit par une variation de la pénétrance liée à la sélection de gènes modificateurs dans un sens différent suivant 1'orientation des races (LAUVERGNE et al., I963), soit en considérant le "gène culard " lui-même et en invoquant une variation de la fréquence des hétérozygotes dans les échantillons de parents considérés comme normaux (cf. ci-dessus). La première interprétation explique en particulier les variations de fréquence du caractère culard dans ANONYME (I966), suivant que 1'on considère des femelles de races laitières ou de races à viande; le deuxième explique les résultats de LAVIN ARENAS (I964) : cet auteur obtient en effet dans la descendance de génisses normales issues d'ancêtres mâles culards (père et grand-père) des fréquences d'apparition de veaux culards de $63 \mathrm{p}$. Ioo et $98 \mathrm{p}$. Ioo suivant que les génisses étaient accouplées à des taureaux normaux ou culards. Ce résultat est intéressant car il permettrait d'augmenter la fréquence des veaux culards tout en réduisant les difficultés de reproduction liées à cette anomalie chez les femelles culardes.

Il est intéressant de rappeler en outre les possibilités de modifier la péné- 
trance d'un gène par sélection. KовоzIEFF et al. (I963) étudiant une anomalie chez la souris, 1'hypotrichose périodique due à un gène partiellement récessif, constatent qu'à chaque nouvelle génération la fréquence d'apparition des anormaux dans la descendance de couples constitués par des individus possédant ce gène mais normaux diminue et expliquent ceci en invoquant 1'accumulation de gènes modificateurs inhibiteurs qui réduiraient la pénétrance du gène responsable de l'anomalie. Une expérience semblable en vue d'augmenter la fréquence d'apparition du caractère culard en croisement de première génération est actuellement en cours de réalisation pour les bovins charolais et garonnais.

Reçu pour publication en janvier r97o.

\section{REMERCIEMENTS}

Nous tenons à remercier MM. Vieillart et Masy-Perier qui nous ont procuré les facilités matérielles nécessaires à la réalisation de cette expérience.

Nous exprimons d'autre part notre vive reconnaissance à MM. HANSET, LaUvergne et OLlivier pour les critiques et suggestions qu'ils nous ont émises dans la rédaction de ce mémoire.

\section{SUMMARY}

\section{STUDY OF THE DOUBLE-MUSCLE CARACTER \\ V. - MATINGS BETWEEN DOUBLE-MUSClE AND NORMAL CATTLE}

Studies done on the farm of Charolais cattle to clarify the determinism of the double-miscle character, have brought to light a large variation in the expression of this character as related to environment.

To overcome this drawback, we carried out a factorial mating plan on an experimental farm between double-muscle (C) and normal $(\mathrm{N})$ animals of the two sexes which have a clear defined phenotype. In general, the progeny obtained ( 83 live calves) may be classed in one of these two categories, with no hesitation: few of them presented an intermediate phenotype. The percentages of double-muscle calves were $87.5,10.0,9.5,0.0$, respectively following $C \times C$; $C \times N ; N \times C ; N \times N$ matings. Moreover, the score expressing the double-muscle character seems, on the average, slightly higher for normal calves, out of the $C \times N$ and $N \times C$ matings. It is lower for calves from normal parents.

These results lead us to suppose a monofactorial action with partial dominance and incomplete penetrance. The latter would be at least $10 \%$ in the heterozygous and $78 \%$ in the homozygous. The possibility of increasing penetrance of the character by selection of modifying genes is discussed.

\section{RÉFÉRENCES BIBLIOGRAPHIQUES}

ANonyme, r96r. Muscular hypertrophy or "Double Muscling " in a friesian calf. Rep. Prod. Div. Milk Mktg. $B d$. (II), I22-124.

ANONYME, I966. Document de travail sur les recherches concernant le caractère culard. Journées Étud. Fed. Eur. Zoot., Edimbourg, 35 p. ronéoté.

Hanset R., r96r. Le problème des bovins "à croupe de poulain ". Annls. Med. Vét., 105, I4-3I.

HANSET R., 1967. Le problème de l'hypertrophie musculaire ou caractère culard dans la race bovine de Moyenne et Haute Belgique. Annls. Med. Vet., 111, 140-1 80.

KAISER, I888. Ueber die sogenannten doppellendigen Rinder. Landw. Jbr, 17, 387-403. 
Kobozieff N., Pomriaskinsky-Kobozieff N., Gemaming E., rg63. Hypotrichose périodique chez la souris. Annls. Biol. anim. Bioch. Biophys., 3, 207-21 8.

I,A Guterivière M. DE, I964. Le caractère culard dans la race charolaise: son expression, ses variations. Mém. fin Étud. Inst. Techn. Prat. Agric., Paris, iा6 p., ronéoté.

I auvergne J. J., Vissac B., Perramon A., I963. Étude du caractère culard. I. Mise au point bibliographique. Annls. Zootech., 12, I35-156.

I,Auvergne J. J., Boyazoglu J. G., HubFrt D., I968. L,e phénomène culard chez les bovins : bibliographie annotée. Bull. tech. Dép. Génét. anim. (Inst. natn. Rech. Agron., Fr.), nº 2.

I.AVIn ARenas D. S., r964. La raza Asturiana de los Valles como productora de carne. Notic. Neosan., 122, $6 \mathrm{I}-87$.

PACI C., I935. Indagini e controlli sulla formazione della sottorazza albese. Riv. Zootech., 12, I49-I59.

Ramoṇt R., 1957. Studio sui bovini piemontesi a groppa doppia ». Annali Accad. Agric., Torino, 99, $60 \mathrm{p}$.

RAmond R., I965. Risultati di una prova di allevamento di bovine piemontesi del tipo " della coscia ע. Annal. Sper. Agr., 17, 47I-489.

Rollins W. C., Julian I. M., Carrol F. D., I968. Double muscle segregants in an inbred line of $A$ berdeen Angus cattle. XII Int. Congr. Genet., 1, 276.

VISSAC B. I.AUVERGNe J. J., I969. Finquête sur le caractère culard dans la zone charolaise (abstr.). Annls. Génét. Sel. anim., 1, I77.

Vissac B., Perreau B., Menissier F., Mauleon P. Étude du caractère culard. ViI. Bilan d'exploitation d'un troupeau de femelles. Annls. Génét. Sel. anim. (à paraître).

WEBER A. D., IBSEN H. L., r934. The occurence of the double-muscle character in purebred beef cattle. Proc. Am. Soc. Anim. Prod., 228-232.

Wriedt C., r929. Die Vererbung des Doppellender Characters bei Rindern. Z. indukt. Abstramm.-u. Vererb Lebre, 51, 482-486. 